\title{
MULTIPLE ANEURYSMS IN A PATIENT WITH TAKAYASU'S ARTERITIS ASSOCIATED WITH MYASTHENIA GRAVIS AND THYMOMA
}

Adriane Souza da Paz ${ }^{1, \star}$, Victor Pereira Mattos ${ }^{1}$, Ana Luisa Souza Pedreira ${ }^{1}$, Mittermayer Barreto Santiago ${ }^{1}$

1.Universidade Federal da Bahia, Salvador (BA), Brazil.

${ }^{\star}$ Corresponding author: adriane.spaz@hotmail.com

\section{BACKGROUND}

Multiple aneurysms are an uncommon manifestation of Takayasu's arteritis (TA) and may predict greater severity due to the risk of rupture. The association of TA and myasthenia gravis (MG) is unusual, and, to our knowledge, this is the first case report in the literature with such association.

\section{CASE REPORT}

A 47-year-old woman was admitted at a university hospital to investigate dyspnea and abdominal pain. She was diagnosed with TA at 23 years because of resistant hypertension due to the right renal artery stenosis requiring a surgical approach. A histopathological study revealed thickening of the middle arterial layer with an inflammatory process rich in lymphocytes, suggesting TA. She was not treated for her vasculitis and remained asymptomatic during the following years. At 39 years, she was diagnosed with MG due to palpebral ptosis and muscle weakness. She was treated with neostigmine and azathioprine for four years and remained asymptomatic since then. On admission, laboratory tests demonstrated an erythrocyte sedimentation rate of $54 \mathrm{~mm}$ and C-reactive protein within the normal range. Transthoracic echocardiogram showed significant left ventricle concentric hypertrophy and dilation of both atria. Abdominal computed tomography (CT) angiography showed focal occlusion of the celiac trunk, total occlusion of the left renal artery, multiple aneurysms in the aorta, and distal right common iliac artery (Fig. 1). There was no wall thickening, or contrast enhancement suggesting vasculitic activity. Thoracic CT angiography revealed a significant intrathoracic mass suggestive of thymoma (Fig. 2). She underwent right renal artery angioplasty with a return of $80 \%$ of the circulation. It was decided not to initiate immunosuppressive treatment, and the vascular surgery team has followed her up for a posterior approach of the aneurysms.

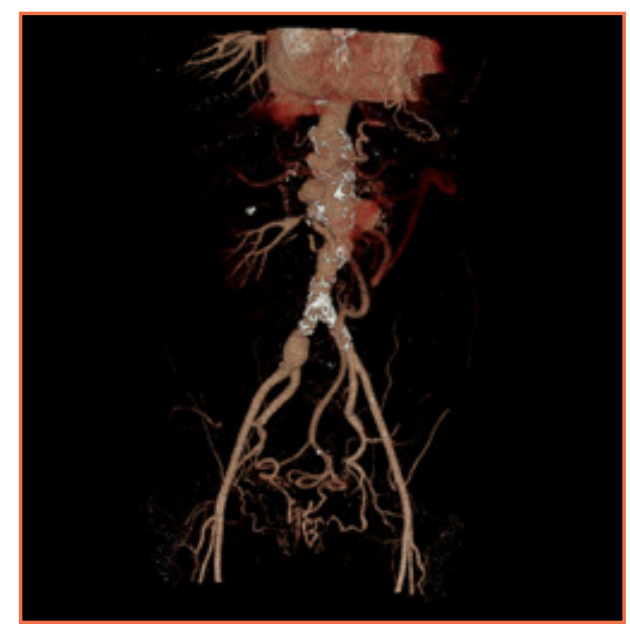

Figure 1. Multiple aneurysms (four saccular dilations in the suprarenal abdominal aorta and one fusiform aneurysm in the distal right common iliac artery).

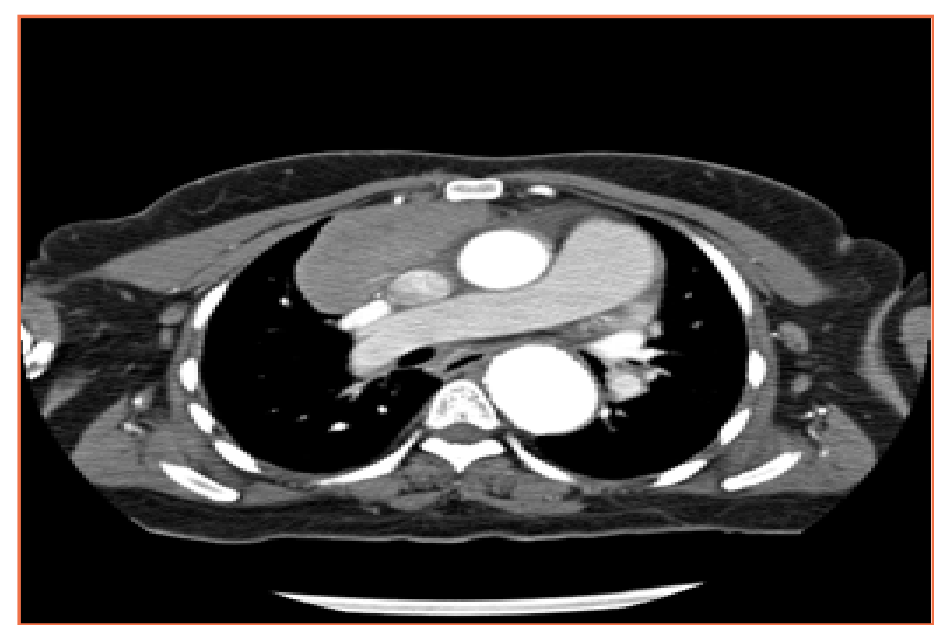

Figure 2. Intrathoracic mass suggestive of thymoma.

\section{CONCLUSION}

Although most TA cases present only arterial stenosis, isolated aneurysms can appear in up $30 \%$ of the patients due to the weakening of the vessel wall poststenotic lesions and increased blood pressure. The appearance of multiple aneurysms, as demonstrated in this case, is uncommon. Furthermore, this is the first case reported in the literature of the association of TA, MG, and thymoma, which seems to be coincidental. 